\title{
Colonic stricture secondary to blunt abdominal trauma- report of a case and review of the aetiology
}

\author{
B.R. Davidson and N.W. Everson \\ Department of Surgery, Leicester Royal Infirmary, P.O. Box 65, Leicester LE2 7LX, UK.
}

\begin{abstract}
Summary: A 24 year old man sustained multiple injuries including blunt abdominal trauma in a motor cycle accident. Five months following the accident he developed a large bowel obstruction. Barium enema examination revealed a stricture of the sigmoid colon which, following surgical resection, was found to consist of a fibrous stenosis. The aetiology of such strictures following blunt abdominal trauma is likely to be a combination of mural haemorrhage and ischaemia.
\end{abstract}

\section{Introduction}

A fibrous stricture may rarely occur in both the colon and small bowel as a late complication of blunt abdominal trauma. ${ }^{1,2}$ Earlier in the post trauma period obstructive symptoms may present due to the formation of an intramural haematoma. Here we present a case of sigmoid colon stricturing which required surgical intervention 5 months post trauma. Symptoms of intestinal obstruction presented in the first week post trauma, settled spontaneously and recurred several months later. This sequence has important implications as to the aetiology of fibrous cicatricial stenosis following trauma. The left colon has been involved more frequently than the right in the few published cases. The majority of left sided lesions have occurred within the descending colon. This case and two previously described involve the sigmoid colon. ${ }^{2,3}$

\section{Case report}

A previously healthy 24 year old man sustained a motor cycle accident. On transfer to the local hospital he was found to be profoundly shocked and cyanosed. Multiple superficial lacerations were present on his face and limbs and a deep penetrating wound on his right anterior chest wall. Surgical emphysema was present over his right hemithorax. Abdominal examination revealed a non-distended abdomen with slight left iliac fossa tenderness. There were no external signs of abdominal trauma and bowel sounds were normal. $\mathrm{X}$-rays of skull, cervical spine, abdomen and pelvis were normal. Facial views showed a LeFort 1 max-

Correspondence: B.R. Davidson, F.R.C.S., Department of Surgery, University College, London WCl, UK.

Accepted: 9 April 1987 illary fracture. A large right sided pneumothorax was present on chest X-ray. He was resuscitated with intravenous fluids and an intercostal drain was inserted. His conscious level improved and he became haemodynamically stable. Two days later he was transferred to the Intensive Care Unit at the Leicester Royal Infirmary.

He was fully conscious and rational and gave no complaint of abdominal pain. Abdominal examination revealed a soft slightly distended abdomen with localized tenderness in the left iliac fossa. Bowel sounds were normal. Abdominal X-ray revealed moderately distended small bowel loops with a few fluid levels. He was treated conservatively with intravenous fluids and nasogastric aspiration. There was minimal aspirate. Over the subsequent two days his abdominal tenderness and distension settled. He was commenced on oral feeding and passed a normal bowel motion. One week following initial injury he was transferred to the care of the maxillo-facial surgeons. His maxillary fractures were reduced and he was discharged home one week later with no further complaint of abdominal pain. When seen two months following initial injury he complained of occasional episodes of left iliac fossa pain worsened by coughing or opening bowels. There were no significant findings on clinical examination. Two weeks later he was asymptomatic.

Five and a half months following the initial trauma he was readmitted to the Leicester Royal Infirmary complaining of severe abdominal pain. Episodes of lower abdominal colic associated with abdominal distension had been increasing in frequency and severity since his discharge from follow-up. Abdominal examination revealed a distended nontender abdomen with hyperactive bowel sounds. 


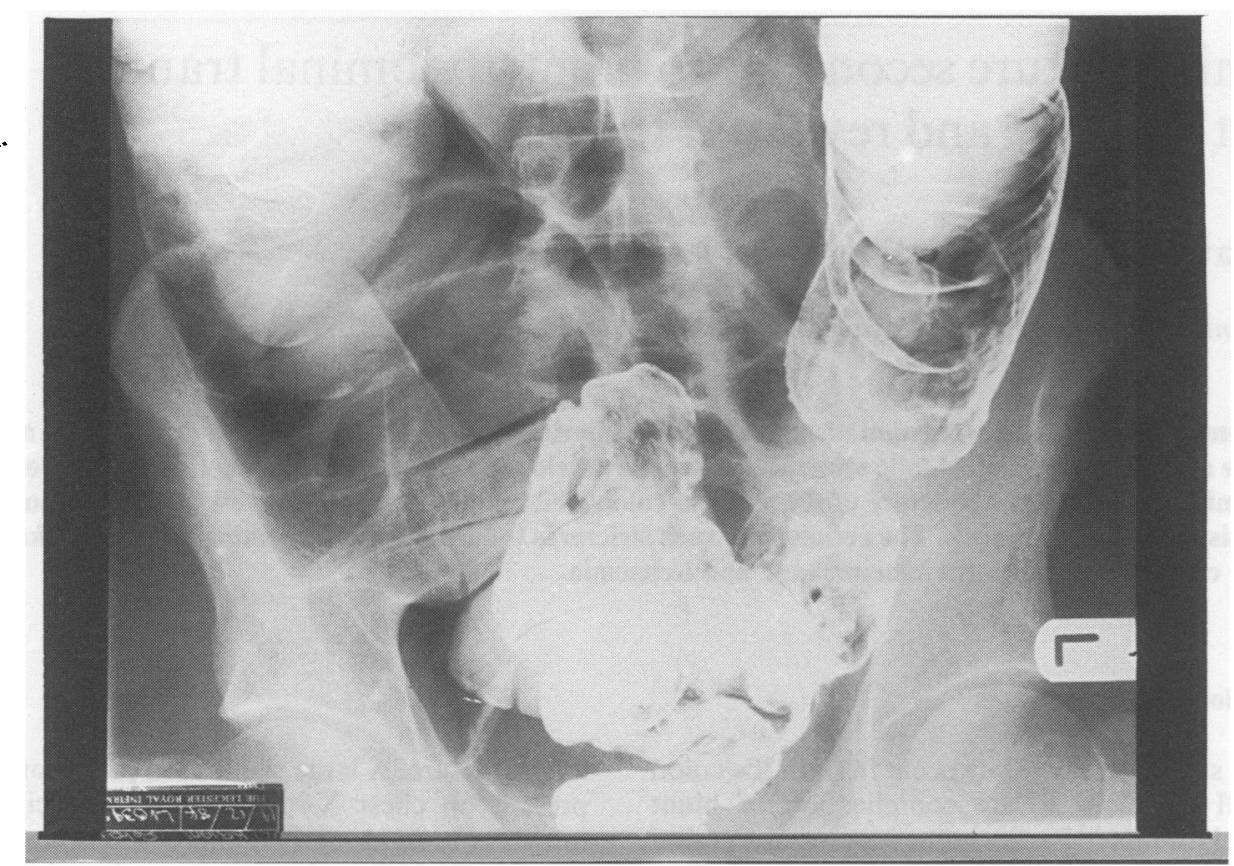

Figure 1 Barium enema X-ray showing localized stricture of the sigmoid colon.

Gaseous distension of the colon and several small bowel loops were present on abdominal X-ray. Barium enema revealed an irregular stricture of the upper sigmoid colon with proximal colonic distension compatible with carcinoma or Crohns' disease (Figure 1). Following evacuation of the barium a flexible sigmoidoscopy was carried out; at $60 \mathrm{~cm}$ the lumen was irregularly narrowed preventing passage of the instrument. Mucosal biopsies at this level were normal. Conservative treatment produced no improvement in his symptoms or signs and laparotomy was therefore carried out.

At laparotomy a firm mass was present in the upper sigmoid colon which was firmly adherent to the lateral abdominal wall. Proximally the colon was moderately distended. Two loops of small bowel, also significantly distended, were fixed to the colonic mass. The small bowel loops were freed and oversewn and the sigmoid mass resected with end-to-end anastomosis in two layers. Post-operative progress was uneventful and he was discharged home well one week later. No further problems have occurred.

Histology of the resected specimen showed a tight stricture of the sigmoid colon. There was loss of surface mucosa with destruction of glands. The lamina propria was congested and contained stromal haemorrhages. The majority of the bowel wall was replaced by dense fibrous tissue.

\section{Discussion}

The variation in the forms of causative trauma would suggest that the left sided predominance of stenotic colonic lesions is predisposed to on an anatomical basis. ${ }^{4}$ This could be explained by the space occupying nature of the liver leaving a reduced area of susceptible colon. Liver lacerations not infrequently result from blunt abdominal trauma. ${ }^{5}$ There is also a significant difference in the anatomy of the vascular supply to the right and left colon, ischaemic lesions of the colon principally affecting the left colon.

A number of theories about the aetiology of fibrous stenotic colonic lesions have been suggested. The formation of a traumatic intramural haematoma was suggested by Altner ${ }^{2}$ in 1964 as a predisposing factor in the formation of a fibrous stricture. This, however, would seem unlikely to lead to a cicatricial stenosis. Studies carried out on traumatized rat colon by Mays \& Noer in $1966^{4}$ showed that solitary mural haematomas tended to resolve completely with no fibrous tissue formation. Complete ischaemia of the colon, as would occur with mesenteric damage, would seem an unlikely predisposing factor to late colonic stenosis as a clinical state suggestive of blood loss, toxaemia or bowel perforation would present early. This would tend to occur before adhesions would allow the formation of a chronic lesion. 
A similar explanation would tend to make a colonic perforation an unlikely primary event, although the dense adhesion to the abdominal wall and small bowel could be interpreted as an attempt at localization. Mays \& Noer ${ }^{4}$ also suggested a loss of surface mucosa with bacterial invasion as a possible causative factor in fibrous tissue reaction. This, however, was not supported by their own rabbit colon experimentation, which showed that rabbit colon denuded of mucosa did not form a fibrous stricture but merely reformed its mucosal covering. This explanation, furthermore, overlooks the fact that the majority of lesions are found with intact mucosa at the site of stricture.

\section{References}

1. Shively, E., Pearlstein, L., Kinnaird, D.W. et al. Posttraumatic intestinal obstruction. Surgery 1976, 79: 612617.

2. Altner, P.C. Constrictive lesions of the colon due to blunt trauma to the abdomen. Surg Gynecol Obstet 1964, 118: $1257-1262$.

\section{Conclusion}

It seems that no single pathological process produces a fibrous colonic stricture several months following abdominal trauma. The primary crushing event probably produces oedema, mural haemorrhage and ischaemia of the colon with resultant replacement of the muscularis with fibrous tissue. The incomplete nature of the ischaemia prevents the vivid presentation of infarcted bowel.

Fibrous stricturing should be considered in the differential diagnosis of abdominal colic or intestinal obstruction where a history of blunt abdominal trauma is obtained.

3. Moell, P.O. Posttraumatisk Colonstriktur. Nord Med 1958, 60: $1834-1835$.

4. Mays, E.T. \& Noer, R.J. Colonic stenosis after trauma. J Trauma 1966, 6: 316-331.

5. Howell, H.S., Bartizal, J.F. \& Freeark, R.J. Blunt trauma involving colon and rectum. J Trauma 1976, 16: 624-632. 